\title{
评 述庆祝中国科学院兰州化学物理研究所成立60周年专刊
}

\section{低共熔溶剂在样品前处理中的应用}

\author{
韩晓菲 ${ }^{1,2}$, 陈佳 ${ }^{1}$, 张海娟 $^{1}$, 李湛 $^{1}$, 邱洪灯 ${ }^{1 *}$ \\ 1. 中国科学院兰州化学物理研究所, 中国科学院西北特色植物资源化学重点实验室/甘肃省天然药物重点实验室, 兰州 730000 \\ 2. 中国科学院大学, 北京 100049 \\ *通讯作者, E-mail: hdqiu@licp.cas.cn
}

收稿日期：2018-08-23; 接受日期：2018-10-09; 网络版发表日期：2018-12-04

国家自然科学基金(编号: 21475142, 21822407)、甘肃省杰出青年科学基金(编号：1506RJDA281)和中国科学院兰州化学物理研究所“一三五” 战略规划重点项目资助

\begin{abstract}
摘要在化学分析过程中, 为了浓缩复杂样品中的微量分析物, 去除基质千扰, 通常需要进行样品前处理, 开发 一种简单、高效、快速且环境友好的样品前处理技术至关重要. 低共熔溶剂(DESs)作为一种新兴的绿色溶剂, 与 传统有机溶剂相比, 具有低挥发性、可生物降解、环境友好、成本低和组合灵活等特点, 可结合各种萃取技术如 超声辅助萃取、微波辅助萃取、中空纤维萃取和固相萃取等, 广泛应用到样品前处理中. 该技术不仅提高了萃取 效率, 而且可以降低常规分析的成本, 减少对人类健康和环境污染影响. 本文综述了近5年DESs在食品、环境、 生物、天然产物以及石油化工等领域的样品前处理过程中的应用.
\end{abstract}

关键词低共熔溶剂(DESs), 样品前处理, 萃取, 化学分析

\section{1 引言}

在分析复杂样品基质中的微量物质时，样品前处 理是必不可少的环节. 合适的样品前处理技术，不仅 可以有效地富集微量的目标分析物，而且能够消除样 品基质中的干扰物，降低对仪器分析系统的损坏，从 而提高方法的灵敏度，降低检测限. 传统的样品前处 理方法操作繁琐耗时且有机溶剂用量大，污染环境， 危害身体健康. 因此，发展高效、快速、准确、灵 敏、环境友好的样品前处理技术成为现代分析技术的 一个重要发展方向.

低共熔溶剂(DESs)通常是由一定化学计量比的氢 键受体(如季铵盐) 和氢键给体(如羧酸、醇、胺类和糖
类等)组合而成的低共熔混合物 ${ }^{[1 \sim 3]}$. 作为一种新兴的 绿色溶剂, DESs的制备简单, 成本低, 无需复杂的纯化 步骤 $^{[4,5]}$, 已经广泛应用于电化学 ${ }^{[6 \sim 8]}$ 、有机反应 ${ }^{[9 \sim 12]}$ 和 功能材料 ${ }^{[13 \sim 15]}$ 等领域. 这种新颖的溶剂具有高热稳定 性、低导热率、低挥发性, 可与先进的分离技术(高效 液相色谱或气相色谱技术)结合, 近年来其在分离分析 领域的应用层出不穷 ${ }^{[16]}$.

$\mathrm{DESs}$ 可作为萃取剂结合各种萃取技术(超声辅助 萃取、微波辅助萃取、中空纤维萃取和固相萃取等) 应用在样品前处理中, 主要依赖于以下 4 个方面的物化 性质 ${ }^{[16]}$ : (1) 凝固点依赖于DESs的组成及其摩尔比; (2) 通常情况下, 密度比水高, 在分散液液微萃取 (DLLME)中能够快速沉降, 实现两相分离; (3) 室温下

引用格式: Han X, Chen J, Zhang H, Li Z, Qiu H. Application of deep eutectic solvents in sample preparation. Sci Sin Chim, 2018, 48: 1548-1560, doi: 10.1360/ N032018-00192 
相对较高的黏度，在单滴微萃取中促进针尖处较大液 滴的悬浮; (4) 不同组分合成的DESs 具有不同极性, 从 而提高了对不同极性分析物的萃取效率. 与传统的有 机溶剂相比, DESs 具有低毒性、可生物降解、环境友 好、易再生等优点，为其在样品前处理领域的应用提 供了较大空间. 本文对近年来DESs在食品、环境、生 物样品、天然产物以及石油化工分析中的应用进行了 总结与评述.

\section{DES在食品分析中的应用}

农产品中的农药残留、乳制品中的抗菌素、饮用 水中的重金属离子和食用植物油中的植物激素等对人 体健康具有潜在危害. 因此, 应将这些食品中有害物质 的量控制在安全范围内. 从这个角度来看, 发展快速、 准确、灵敏、经济和环境友好的分析方法用于萃取和 检测食品中这些化合物非常重要.

Farajzadeh等 ${ }^{[17]}$ 发展了一种基于DES的气体辅助 液相微萃取(GA-LPME)的方法，结合气相色谱 $(\mathrm{GC})$ 检 测了水果和蔬菜样品中的农药残留. 以摩尔比为 $1: 2$ 的 氯化胆碱和4-氯苯酚制得的DES加入到含分析物的样 品溶液中进行萃取. 为使分析物更好地萃取到DESs的 液滴中, 空气被鼓泡加入其中, 从而形成浑浊的溶液. 离心之后，下层沉降部分进入 $\mathrm{GC}$ ，利用火焰离子检测 器(FID)进行分离检测. 该方法取得了与其他农药残留 检测方法 ${ }^{[18,19]}$ 水平相当的检测限和线性范围，而且操 作简单、成本低、萃取时间短、效率高.

$\mathrm{Li}$ 等 ${ }^{[20]}$ 用摩尔比为 $1: 2$ 的氯化胆碱和丙三醇制备 了DES, 并将其分别应用到两种不同的萃取方法中, 实 现了对牛奶中氯霉素和甲砜霉素的纯化. 在DLLME 中, DES与三氯甲烷作为萃取剂, 将其以不用的比例 混合进行优化，在2:1的比例时取得了最高的萃取效 率. 在固相萃取方法中，制备了DESs修饰的分子印迹 聚合物(DESs-MIPs，氯霉素和甲砜霉素两个模板)，用 于牛奶中两种抗菌素的萃取，并与制备的分子印迹聚 合物(MIPs，无DESs修饰)和非印迹聚合物(NIPs，无模 板)进行比较，DESs-MIPs取得了最高的回收率. 在两 种萃取方法的比较中, DESs-MIPs的固相萃取法的回 收率也优于DLLME.

Zounr等 ${ }^{[21]}$ 采用基于DES超声辅助液相微萃取技 术萃取富集了几种食品和水中的镉离子，通过电热原
子吸收光谱法(ETAAS)进行测定. 他们合成了基于氯 化胆碱的几种不同的DESs, 经过条件优化, 其中以摩 尔比为 $1: 4$ 的氯化胆碱和苯酚合成的DES在 $\mathrm{pH} 4$ 的条 件下具有最佳的萃取效果. 在萃取过程中加入四氢呋 喃(THF，非质子性溶剂)作为乳化剂，使得DES液滴在 水/四氢呋喃中不溶, 通过离心即可实现两相的分离. 与传统的方法相比, 该方法成本低, 萃取过程简单, 环 境友好, 而且取得了较高的富集因子、灵敏度和较低 的检测限.

$\mathrm{Tan}$ 等 ${ }^{[22]}$ 制备了一系列不同比例的DESs 并用于食 用植物油中植物激素(吲哚-3-乙酸、吲哚-3-丁酸和4碘苯氧基乙酸)的萃取. 不同类型的DESs由不同的氢 键给体和氢键受体组成, 摩尔比为 $1: 3$ 的四甲基氯化铵 和乙二醇制备的DES显示出最佳的萃取效率, 且在10 $50 \mathrm{~mL}$ 范围内, $30 \mathrm{~mL}$ DESs获得了最高的效率. 在 $50^{\circ} \mathrm{C}$ 下超声 7 min即可达到萃取平衡. 该方法简化了检测食 用植物油中痕量植物激素的前处理步骤.

植物酚类物质是植物第二大代谢物, 广泛存在于 各类植物中. 由于这些酚类物质具有抗氧化性, 能够 预防各类与氧化应激相关的疾病, 得到了越来越多的 关注. 因此，近几年从植物以及食品中萃取检测这类 化合物已经成为生命健康领域的研究热点.

Bubalo等 ${ }^{[23]}$ 用一种基于氯化胆碱的DES萃取葡萄 皮中的酚类物质。该方法制备的DES中用含有 $25 \%$ 水 的甲酸作为氢键给体. 与微波辅助萃取(MAE) 和传统 方法相比, 超声辅助萃取(UAE)方法取得了最佳的萃 取效果. 他们提出, 在用DES萃取酚类化合物之前, 酚 类化合物在DES中的稳定性应该被研究.

García等 ${ }^{[24]}$ 用氯化胆碱和糖类、醇类以及尿素以 不同比例混合制备的DESs对初櫵橄榄油中的酚类物 质进行萃取. 与传统的方法(甲醇/水)相比, 在他们的萃 取条件下, 所研究的DESs提高了对橄榄油中酚类化合 物的萃取效率, 有力地说明了DESs 是一种极好的萃取 溶剂, 对不同极性的酚类化合物具有良好的溶解度. DESs作为一种更绿色、高效的萃取溶剂可以代替甲 醇从橄榄油中大规模萃取酚类化合物. Paradiso等 ${ }^{[25]}$ 用 基于葡萄糖和乳酸的DES萃取特级初椥橄榄油中的酚 类物质, 并采用光谱方法检测, 橄榄油中整个酚类物质 的含量与萃取物的光谱性质相关. 在几个波长下测量 DES萃取物的吸收就可以筛选橄榄油中整个酚类物质 的含量, 极大地减少了有毒溶剂的使用. 
Khezeli等 ${ }^{[26]}$ 用一种简单灵敏的基于DES的超声 辅助液液微萃取(UA-LLME)方法结合高效液相色谱紫外检测器(HPLC-UV)检测了植物油中的阿魏酸、咖 啡酸和肉桂酸(图1). 将摩尔比为 $1: 2$ 的氯化胆碱和乙二 醇制备的DES加入到溶解在正己烷的样品中，然后将 此混合物超声 $5 \mathrm{~min}$, 形成含微滴的乳状液, 从而增加 在DES和样品之间的接触面积. 萃取后, 富含分析物的 DES相通过离心与正己烷分离, 再进入HPLC-UV分析. 该方法取得了较低的检测限、较高的回收率和良好的 重现性.

此外, DESs在食品分析方面的应用还可参见 表 $1^{\text {[28 35] }}$.

\section{DES在环境分析中的应用}

由于人类活动的影响，很多有机和无机的污染物 会进入到环境水(河水、湖水和海水)和土壤中, 进入 生态系统后通过生物富集进入人体，影响身体健康. 因此，发展一种有效的分析方法检测这些污染物具有 重要意义.

Zarei等 ${ }^{[36]}$ 首先制备了基于钠蒙脱石的磁性纳米
颗粒, 再将其分散到以薄荷脑为氢键受体, 不同羧酸为 氢键给体制备的DESs中，从而制得一种基于DES的铁 磁流体, 结合直接分散液滴微萃取的方法, 借助HPLC$\mathrm{UV}$ 检测了水和土壤中的爆炸物. 所制备的铁磁流体具 有较强的吸附能力和疏水性, 可以稳定地分散在水中 而不会沉降. 将该磁性流体的微滴加入到搅拌的样品 溶液中完成萃取, 再通过磁铁即可分离. 经过优化影 响萃取效率的各种因素之后实现了高效的萃取.

Farajzadeh等 ${ }^{[37]}$ 在液液微萃取步骤中原位合成 DES萃取水样中的几种多环芳烃, 后利用HPLC-二极 管阵列检测器(DAD)检测. 在含多环芳烃的水样中加 入摩尔比为 $1: 2$ 的氯化胆碱和对氯苯酚, 先振荡成均匀 的溶液, 后加热反应生成DES后成浑浊状态, 即可完成 萃取过程. 之后, 溶液进行离心, 下层沉降部分进入 HPLC-DAD分析. 该DES与水不互溶, 在水溶液中合 成，同时完成PAHs的萃取. Khezeli等 ${ }^{[38]}$ 将基于DES的 乳化液液微萃取(ELLME-DES)方法用于水样中苯、 甲苯、乙苯 $(\mathrm{BET})$ 和7种多环芳烃的萃取. 氯化胆碱与 苯酚以摩尔比 $1: 2,1: 3$ 和 $1: 4$ 混合制备DESs, 苯酚作为 氢键给体与目标分析物有强的 $\pi-\pi$ 相互作用. $100 \mu \mathrm{L}$ 的 DES加入到 $1.5 \mathrm{~mL}$ 含目标分析物的样品溶液中, 立即

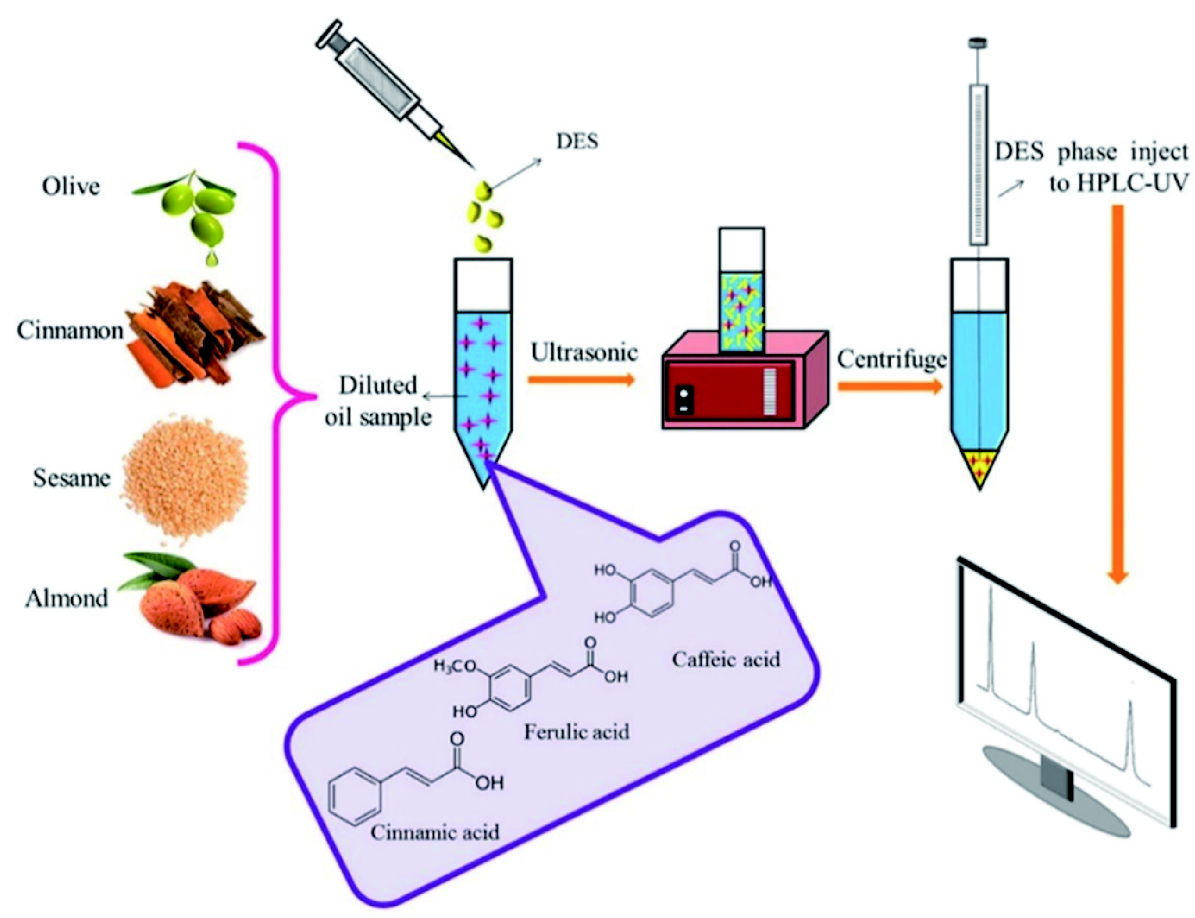

图 1 一种绿色的基于DES的超声辅助液液微萃取方法结合HPLC-UV检测植物油中的3种酚酸类物质(网络版彩图)

Figure 1 A green UA-LLME based on DES for the HPLC-UV determination of three phenolic acids from vegetable oils (color online). 
表 1 总结DESs在样品前处理中的应用(2013 2018年)

Table 1 Overview applications involving deep eutectic solvents (DESs) in sample preparation (2013-2018)

\begin{tabular}{|c|c|c|c|c|c|c|}
\hline 分析领域 & 分析物 & 样品基质 & DES (摩尔比) & 萃取方法 ${ }^{\mathrm{a})}$ & 分析技术 ${ }^{b)}$ & 参考文献 \\
\hline \multirow{9}{*}{ 食品分析 } & 叔丁基对苯二酚 & 食用油 & 氯化胆碱:乙二醇, $1: 2$ & UALLME & HPLC-UV & {$[27]$} \\
\hline & 铅 & 食品和环境水样 & 氯化胆碱:苯酚, 1:4 & AA-LPME & GFAAS & {$[28]$} \\
\hline & 姜黄素 & 食品和花草茶 & 氯化胆碱:苯酚, 1:4 & VA-ELLME & UV-Vis & [29] \\
\hline & 钒 & 食品 & 氯化胆碱:苯酚, 1:4 & UALLME & ETAAS & [30] \\
\hline & 铝 & 食品和环境水样 & 氯化胆碱:苯酚, 1:4 & UALPME & ETAAS & [31] \\
\hline & 硒 & 食品和环境水样 & 氯化胆碱:苯酚, $1: 3$ & UALPME & ETAAS & [32] \\
\hline & 叔丁基对苯二酚 & 大豆油 & 氯化胆碱:芝麻酚, $1: 3$ & UALLME & HPLC-UV & [33] \\
\hline & 芝麻酚 & 香油 & 氯化胆碱:乙二醇, $1: 2$ & UALLME & HPLC-UV & {$[34]$} \\
\hline & 铅和镉 & 食用油 & 氯化胆碱:尿素, $1: 2$ & LPME & ETAAS & {$[35]$} \\
\hline \multirow{13}{*}{ 环境分析 } & 铅、镉和录 & 土壤和蔬菜 & $\begin{array}{c}\text { 氯化-1-甲基-3-癸基咪唑:十 } \\
\text { 一烷醇, } 1: 2\end{array}$ & DLLME & GFAAS & [47] \\
\hline & $\begin{array}{c}\text { 砷、铬、钼、锑、硒 } \\
\text { 和钒 }\end{array}$ & 农业土壤 & 氯化胆碱:草酸, 1: 2 & UAE & ICP OES & {$[48]$} \\
\hline & 磺胺甲基嘧啶 & 河水 & 氯化胆碱:乙二醇, $1: 2$ & PT-SPE & HPLC & {$[49]$} \\
\hline & 多环芳烃 & 湖水 & 氯化胆碱:乙二醇, $1: 2$ & SPME & UPLC & {$[50]$} \\
\hline & 常见农药 & 环境水样 & $\begin{array}{c}\text { 氯化-1-甲基-3-癸基咪唑:十 } \\
\text { 一烷醇, } 1: 2\end{array}$ & LPME & HPLC-UV & {$[51]$} \\
\hline & 苠基脲 & 环境水样 & $\begin{array}{c}\text { 氯化-1-甲基-3-癸基咪唑:十 } \\
\text { 二烷醇, } 1: 2\end{array}$ & DLLME & HPLC-UV & {$[52]$} \\
\hline & 芳香硝基化合物 & 环境水样 & 氯化胆碱:尿素, $1: 2$ & DSPE & $\mathrm{GC}-\mu \mathrm{ECD}$ & {$[53]$} \\
\hline & 砷 & 环境水样 & 氯化胆碱:苯酚, $1: 3$ & UALPME & ETAAS & {$[54]$} \\
\hline & 磺胺类药物 & 环境水样 & 氯化胆碱:苯酚, $1: 2$ & ELLME & HPLC-UV & {$[55]$} \\
\hline & 苄基脲 & 环境水样 & $\begin{array}{c}\text { 三辛基甲基氯化铵:十二烷 } \\
\text { 醇, } 1: 2.5\end{array}$ & SA-DLLME & HPLC & {$[56]$} \\
\hline & 脂肪酸和安息香酸 & 工业废水 & 氯化胆碱:4-甲酚, 1:2 & USA-DLLME & GC-MS & {$[57]$} \\
\hline & 铬黑T & 废水 & 氯化胆碱:乙醇酸, 1:1 & LLME & UV-Vis & {$[58]$} \\
\hline & 铬 & 环境水样 & 氯化胆碱:乙二醇, $1: 3$ & USA-LLME & ETAAS & {$[59]$} \\
\hline \multirow{5}{*}{ 生物样品分析 } & 铅和镉 & 人类头发、土壤和环境水样 & 氯化胆碱:尿素, $1: 2.5$ & SPE & ETAAS & {$[68]$} \\
\hline & 美沙酮 & 蒸馏水、尿和血浆 & $\begin{array}{c}\text { 氯化胆碱:5,6,7,8-四氢- } \\
5,5,8,8 \text {-四甲基-2-萎酚, } 1: 2\end{array}$ & MSPE & $\begin{array}{l}\text { GC-FID, } \\
\text { GC-MS }\end{array}$ & [69] \\
\hline & 铜和镍 & 环境水样、血清和尿 & 氯化胆碱:尿素, $1: 2$ & SPE & FI-FAAS & {$[70]$} \\
\hline & 采 & 血液 & $\begin{array}{c}\text { 氯化-1-甲基-3-癸基咪唑:十 } \\
\text { 一烷醇, } 1: 2\end{array}$ & VADLLME & GFAAS & {$[71]$} \\
\hline & 铅 & 血液 & 氯化胆碱:尿素, $1: 2$ & HF-LPME & ETAAS & {$[72]$} \\
\hline \multirow{3}{*}{ 天然产物分析 } & 黄酮类物质 & 沙棘叶 & 氯化胆碱:1,4-丁二醇, $1: 3$ & MAE & HPLC & {$[86]$} \\
\hline & $\begin{array}{c}\text { 生物碱、黄酮和 } \\
\text { 儿茶酚 }\end{array}$ & 茶叶 & 氯化胆碱:1,4-丁二醇, $1: 3$ & MCE & UPLC-MS & {$[87]$} \\
\hline & 黄酮类物质 & 槐花 & $L$-脯氨酸:丙三醇, $1: 2.5$ & UAE & LC-UV & {$[88]$} \\
\hline
\end{tabular}

a) UALLME/USA-LLME: 超声辅助液液微萃取; AA-LPME: 空气辅助液相微萃取; VA-ELLME: 浴流辅助乳化液液微萃取; UALPME: 超 声辅助液相微萃取; DLLME: 分散液液微萃取; UAE: 超声辅助萃取; PT-SPE: 管尖固相萃取; SPME: 固相微萃取; DSPE: 分散固相萃取; SADLLME: 盐辅助分散液液微萃取; MSPE: 磁固相萃取; HF-LPME: 中空纤维液相微萃取; MHD: 微波辅助的水蒸馏法; MAE: 微波辅助萃取; MCE: 机械化学提取. b) GFAAS: 石墨炉原子吸收光谱法; ETAAS: 电热原子吸收光谱法; ICP OES: 电感耦合等离子体发射光谱法; UPLC: 超 高效液相色谱法; GC: 气相色谱法; $\mu \mathrm{ECD}$ : 微电子捕获检测器; FI-FAAS: 流动注射的火焰原子吸收光谱法 
形成均一的溶液, 再加入 $100 \mu \mathrm{L}$ THF超声 $20 \mathrm{~min}$ 形成 浑浊的状态. 萃取后, 经过离心分离, DES相进入 HPLC-UV分析检测. 超声使DES完全分散在水相中, 促进微小乳化液滴的形成, 加速传质, 增加目标物与 DES的接触面积. 使用THF (作为乳化剂)降低了水分 子与DES接触的趋势, 促进了DES微滴的自聚. 该方法 获得了良好的线性、精密度和准确性，具有简单、灵 敏和成本低的优势, 同时具有较高的富集因子. Yousefi 等 ${ }^{[39]}$ 将基于 DES的磁性巴基凝胶(DES-MBG)作为传 统挥发性有机溶剂的替代物发展了一种顶空单滴微萃 取(HS-SDME)方法. DES-MBG由氯化胆碱/氯苯酚和 磁性多壁碳纳米管(MMWCNTs)复合物组成，能在几 周之内保持稳定. 磁化率、高黏度、高吸附性、对有 机分析物可调节的萃取性使得制备的凝胶适合作为 HS-SDME的接受相. DES-MBG的磁化率和高黏度使 得微滴具有高稳定性, 从而可以在高温和高转速下进 行萃取. 与传统的HS-SDME相比, 高转速缩短了样品 前处理时间, 提高了萃取效率. 而且, 高稳定性的接受 相使得使用更大的液滴成为可能，从而提高方法的灵 敏度，降低检测限. DES与MMWCNTs的协同效应提 高了萃取效率. 因此，DES-MBG在SDME中是一种有 效的萃取剂. 该方法与气相色谱法结合, 成功地测定了 水和尿液中有害的挥发性有机化合物(苯、甲苯、乙 苯和二甲苯异构体).

Yilmaz等 ${ }^{[40]}$ 发展了一种超声辅助的基于DES的乳 化液相微萃取(UA-DES-ELPME)结合火焰原子吸收光 谱法(FAAS)检测水样中三价和六价铬离子的方法. 作 者制备了几种不同的DESs: (1) 氯化胆碱和苯酚以不 同比例混合; (2) 四丁基氯化铵与癸酸; (3) 甲基三辛基 氯化铵与癸酸, 将其应用到铬离子的萃取中, 通过比较 回收率，最终选择摩尔比为 $1: 3$ 的氯化胆碱和苯酚制备 的DES进行萃取. 超声促进微米和纳米尺寸的团聚体 形成, 加速传质, 提高了萃取效率. 该研究表明, DESs 作为一种绿色的萃取剂可替代传统的挥发性溶剂.

二苯甲酮类物质是一种紫外线吸收剂，广泛应用 于个人护理品、化妆品和药品中 ${ }^{[41]}$, 可通过泳池、浴 场和废水处理厂等途径进入环境中 ${ }^{[42]}$. 在动物身上进 行的毒理学研究已经证明, 一些有机的紫外线吸收剂 即使在微量水平也可能会诱发䧳激素和抗甲状腺作 用 ${ }^{[42,43]}$. Ge 等 ${ }^{[44]}$ 用薄荷脑和癸酸合成了DES并将其用 作气体辅助分散液液微萃取的萃取剂, 富集和萃取水
样中6种二苯甲酮类物质，再结合HPLC-DAD进行检 测. 空气辅助有利于样品溶液中萃取剂良好液滴的快 速形成，无需使用分散剂，约 $1 \mathrm{~min}$ 就可以实现萃取平 衡. 文中推测疏水的分析物(对羟基苯甲酸酯类、多环 芳烃、多氯联苯和多溴二苯醚)或许也可以用这种方 法检测. 同样是对紫外线吸收剂的检测, Wang 等 ${ }^{[45]}$ 则 是采用由甲基三辛基氯化铵和癸酸以不同比例合成的 DESs 结合超声辅助分散液液微萃取的方法, 利用 HPLC-UV检测了游泳池和河水中的 3 种二苯甲酮类物 质. 超声加速良好浑浊溶液的形成, 可以避免使用分散 剂, 明显减少了萃取时间, 提高了萃取效率.

在渔业中，已禁止使用孔雀石绿预防外来寄生虫 和真菌, 但非法使用状况仍然存在. Aydin等 ${ }^{[46]}$ 用摩尔 比为 $1: 4$ 的氯化胆碱和苯酚制备的DES结合超声辅助 的乳化液相微萃取(UA-ELPME), 利用紫外可见分光 光度计检测了鱼类养殖场和水族馆水样中的孔雀石绿 (图2). 在 $\mathrm{pH} 3$ 的水样 (含 $0.3 \mathrm{mg} / \mathrm{L}$ 孔雀石绿)中, 加入 $500 \mu \mathrm{L}$ 合成的DES以及作为乳化剂的四氢呋喃, 超声 $3 \mathrm{~min}$ 后，孔雀石绿被良好分散的DES微滴萃取. 再通 过离心, 使得水相和DES相分离. 在含分析物的DES相 中加入乙醇稀释至 $1 \mathrm{~mL}$ 后, 使用紫外可见分光光度计 (在635 nm波长下)进行检测. 该方法在检测鱼类养殖 场水样中的孔雀石绿上具有较大潜力, 可在日常分析 中作为工业上的分析手段检测鱼类养殖场存在的孔雀 石绿.

此外, DESs在环境分析方面的应用还可参见 表 $1^{[47 \sim 59]}$.

\section{DES在生物样品分析中的应用}

各种各样的方法已经被发展用于从生物样品中萃 取目标分析物, 其中微波酸解法具有快速、成本低和 产生废弃物少的优势, 但是高浓度的酸或氧化试剂不 仅会产生致癌的气体，而且在检测过程中产生基质干 扰. 使用DESs溶解生物样品并萃取目标分析物则可以 有效地避免这些问题, 同时, 可简化操作步骤, 减少分 析时间, 提高萃取效率. Habibi等 ${ }^{[60]}$ 用氯化胆碱和草酸 (1:2, 摩尔比)合成的DES萃取了鱼的肌肉、肝脏和鳃 中的铜、锌和铁, 并利用FAAS进行检测(图3). 在这个 方法中, $100 \mathrm{mg}$ 样品溶解在DES中, $100^{\circ} \mathrm{C}$ 下 $45 \mathrm{~min}$ 后, 加入 $5.0 \mathrm{~mL} 1.0 \mathrm{M}$ 的硝酸溶液. 再经过离心, 取上层清 

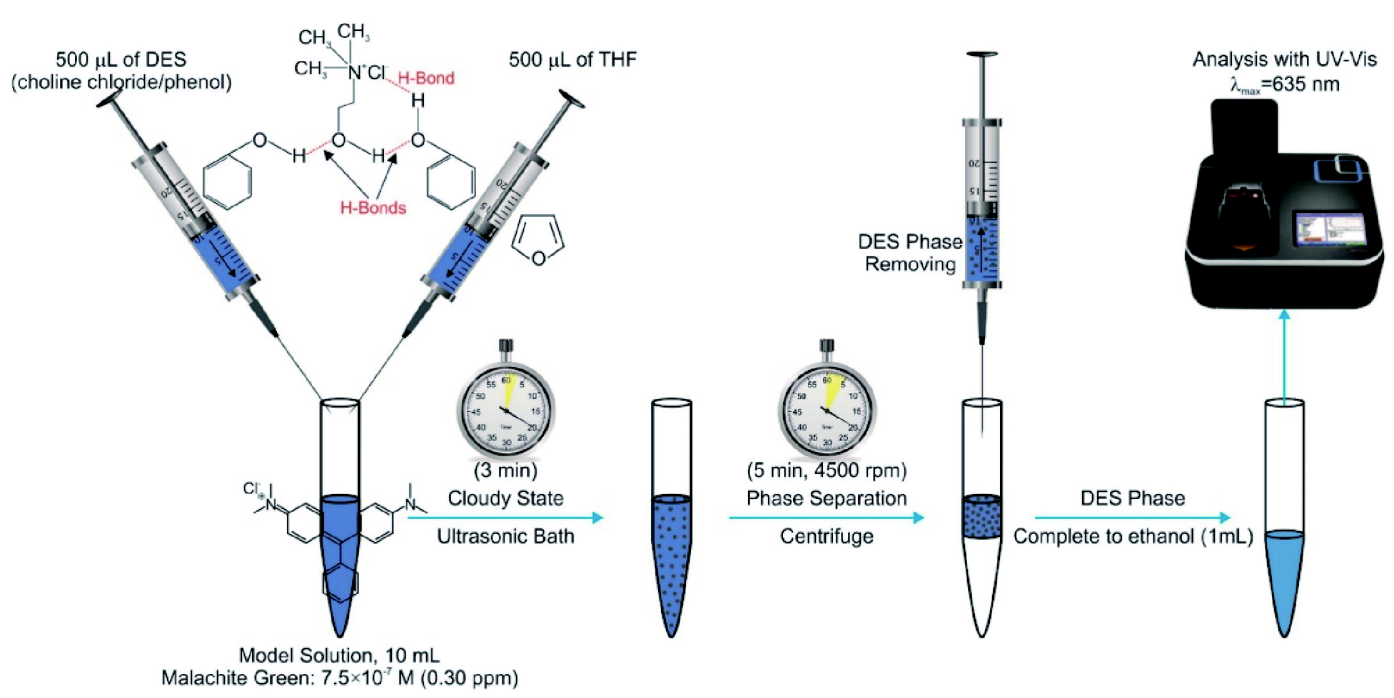

Malachite Green: $7.5 \times 10^{-7} \mathrm{M}(0.30 \mathrm{ppm})$

图 2 一种基于DES的超声辅助乳化液相微萃取方法萃取鱼类养殖场和水族馆水样中的孔雀石绿(网络版彩图)

Figure 2 A DES based UA-ELPME method for malachite green in farmed and ornamental aquarium fish water samples (color online).

液过滤, 稀释到一定体积后, 采用FAAS分析. $\mathrm{HNO}_{3}$ 的 加入提高了铁和铜的回收率, 与传统的酸消化法相比, 明显减少了酸的消耗和萃取时间。该方法简化了实验 步骤, 提高了萃取效率, 避免使用浓酸和氧化试剂, 可 以用于生物样品中金属的常规分析. 相似的, Ghanemi 等 ${ }^{[61]}$ 采用微波辅助的方法将鱼的肌肉和肝脏溶解在 氯化胆碱和草酸 $(1: 2$, 摩尔比)合成的DES中, 萃取铜、

铁、镍和锌离子后，用电感耦合等离子发射光谱法进 行检测。该方法萃取时间短，可检测金属离子的浓度 范围广, 检测限低. Helalat-Nezhad等 ${ }^{[62]}$ 将生物样品(鱼 和藻)完全溶解在氯化胆碱和草酸(1:2, 摩尔比)合成的 $\operatorname{DES}$ 中 $\left(55^{\circ} \mathrm{C}, 45 \mathrm{~min}\right)$, 再加入 $5 \mathrm{~mL}$ 正己烷从DES中萃 取8种多环芳烃，采用HPLC-FL进行检测。与微波辅助 的萃取和加压液体萃取相比, 该方法可以在常压下相 对低的温度下萃取．在最优的条件下，该方法具有良 好的线性范围、检测限和回收率，在日常分析生物样 品中的痕量PAHs中具有较大潜力.

内分泌干扰物是干扰内源性激素常规功能的一类 物质, 由多种化学物质组成, 包括增塑剂、阻燃剂、杀 虫剂、工业副产品、植物化合物的衍生品, 以及甾体 激素等药物 ${ }^{[63]}$. 大多数萃取和检测内分泌干扰物和甾 体激素的方法都集中在环境中的水样, 在生物体液中 的分析较少. Khataei等 ${ }^{[63]}$ 用三相中空纤维液相微萃取 (HF-LPME)的方法萃取了尿液和血浆中的两种甾体激 素(地屈孕酮和醋酸环丙孕酮). 其中, 用摩尔比为 $1: 4$ 的
甲基三苯基磷酸碘化铵和乙二醇合成的DES作为接受 相, 正十二烷作为支持液膜. 甲基三苯基磷酸碘化铵作 为氢键受体, 与分析物有更多的相互作用. 与其他传统 的有机接受相和普通的DESs相比，该DES具有更优良 的性能，由于其低挥发性、低成本和多功能性的特点， 可以将其作为新一代HF-LPME的接受相. 中空纤维具 有良好的样品净化功能, 可以用于复杂样品基质中甾 体激素的萃取.

美沙酮是一种合成的止痛药, 用于阿片类药物(如 海洛因)依赖的脱毒治疗. 发展一种检测生物样本中美 沙酮含量的分析方法是实现最佳治疗的必要条件. Lamei 等 ${ }^{[64]}$ 将DES作为萃取剂应用到空气辅助乳化液 液微萃取中, 结合配有火焰离子化检测器的气相色谱 仪分析了尿液和血液中的美沙酮. 为了得到水溶性的 DES，氯化胆碱和 5,6,7,8-四氢-5,5,8,8-四甲基-2-荎酚 以摩尔比为 $1: 2$ 的比例混合. 将合成的DES和THF (作 为反乳化剂)加入到含分析物的样品溶液中, 形成一种 浑浊的状态. 为了提高溶液的浑浊程度, 分散聚集的 DES液滴到溶液中, 用注射器将溶液快速吸取和注射 10 次. 空气辅助的方法促进了DES在样品溶液中快速 形成良好分散的液滴, 极大地减少了萃取时间.

生物分子在科学研究、制药、工业等领域有广泛 的应用，其中从复杂的混合物中分离出某种特定蛋白 质对了解和揭示某一生命现象和本质有着重要意义. $\mathrm{Xu} \mathrm{u}^{\text {等 }}{ }^{[65]}$ 发展了一种基于 DES 的双水相体系用于蛋白 


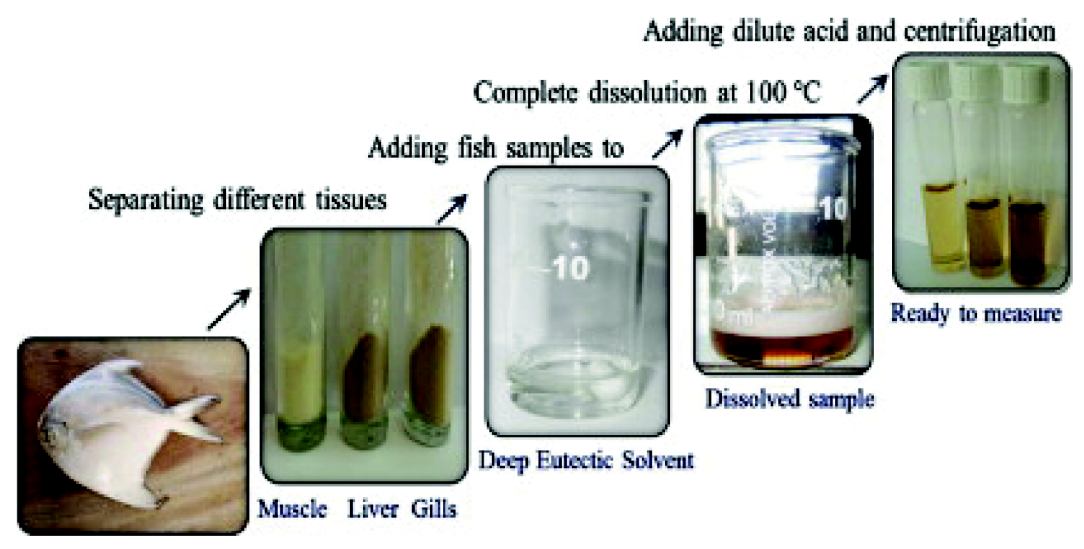

图 3 氯化胆碱和草酸合成的DES萃取鱼样中的铜、铁和锌(网络版彩图)

Figure 3 Based on a choline chloride-oxalic acid deep eutectic solvent for extraction of $\mathrm{Cu}, \mathrm{Fe}$ and $\mathrm{Zn}$ in fish samples (color online).

质的萃取(图4). 将恰当比例的DES、蛋白质和 $2 \mathrm{~mL}$ 磷 酸氢二钾溶液置于离心管中, 在 $25^{\circ} \mathrm{C}$ 下振荡 $20 \mathrm{~min}$ 使 之平衡，然后在 $3000 \mathrm{r} / \mathrm{min}$ 下离心 $5 \mathrm{~min}$. 萃取分离之 后，收集DES相并稀释后通过紫外分光光度计(设定波 长为 $278 \mathrm{~nm}$ ) 检测. 他们合成了 4 种基于氯化胆碱的 DESs用于萃取牛血清白蛋白, 经研究发现, 由氯化胆 碱和丙三醇合成的DES是最合适的萃取剂。该研究表 明，DES-蛋白质团聚体在分离过程中发挥了重要作 用. 通过紫外、红外和圆二色光谱测定，蛋白质萃取 到DES相中，其构象并没有改变. 该方法无毒，原子利 用率和萃取效率高, 但是对牛血清白蛋白的选择性较 差. 此外, $\mathrm{Xu}$ 等 ${ }^{[66]}$ 还发展了一种基于聚低共熔溶剂 (PDES)修饰的磁性微球的磁固相萃取(MSPE)法用于 牛胰腺粗提物中胰蛋白酶的萃取. 修饰PDES后的磁性 微球, 具有良好的化学和热力学稳定性, 较大的比表面 和可调的结构, 表现出了更高的萃取能力和选择性, 循 环使用6次后，萃取能力没有明显损失，而且萃取后的 胰蛋白酶仍保持活性. 该方法在纯化生物分子方面表 现出了巨大潜力. Huang 等 ${ }^{[67]}$ 也利用一种基于DES的 MSPE法实现了对蛋白质(牛血清白蛋白、卵清蛋白、 牛血红蛋白和溶菌酶)的萃取. 将氯化胆碱和葡萄糖合 成的DES修饰在磁性氧化石墨烯 $\left(\mathrm{Fe}_{3} \mathrm{O}_{4} @ \mathrm{GO}\right)$ 表面制 备得到 $\mathrm{Fe}_{3} \mathrm{O}_{4} @ \mathrm{GO}-\mathrm{DES}$. 与 $\mathrm{Fe}_{3} \mathrm{O}_{4} @ \mathrm{GO}$ 相比, $\mathrm{Fe}_{3} \mathrm{O}_{4} @$ GO-DES对酸性蛋白质具有更高的萃取能力. 该方法 合成的DES原子利用率达到了 $100 \%$ ，磁性材料也可以 回收, 但遗憾的是, 回收后磁性材料的萃取能力明显下 降. 即便如此, 该方法制备的 $\mathrm{Fe}_{3} \mathrm{O}_{4} @$ GO-DES成功地应 用到磁固相萃取蛋白质中. 表 $1^{[68 \sim 72]}$.

此外, DESs在生物样品分析中的应用还可参见

\section{DES在天然产物分析中的应用}

传统的有机溶剂被广泛应用在从天然产物资源中 萃取生物活性化合物. 但是, 大量有机溶剂的使用不仅 会在提取物中有残留还会造成环境污染. 而DESs在萃 取生物活性分子方面具有很多优势，如DESs的合成步 骤简单、毒性小、廉价和具有生物相容性等. DESs与 生物活性分子之间氢键相互作用提高了萃取效率, 而 且一些性质不稳定的生物活性分子可以在DESs中稳 定存在.

Dai 等 ${ }^{[73]}$ 合成了 7 种具有不同极性、黏度、组成和 溶解性的天然低共熔溶剂(NADES), 并用其萃取了红 花中不同极性的酚类代谢产物. 与传统溶剂相比, 高 黏度的NADES导致了较低的传质从而影响萃取效率. 该研究通过加入一定量的水, 提高温度和机械搅拌的 方式降低其黏度. 其中, NADES中水的含量对酚类化 合物的萃取影响最大. 经过条件优化, NADES对极性 和低极性的酚类代谢物的萃取能力强于传统的溶剂, 可能是NADES富含羟基、羧基和氨基等基团与酚类 化合物之间的氢键相互作用. 从NADES回收的大多数 酚类化合物回收率为 $75 \%$ \% $97 \%$. 研究表明, NADES具 有从天然的资源中萃取生物活性物质的潜在应用. NADES与目标分析物之间的氢键相互作用不仅提高 了萃取效率, 还提高了目标分析物在NADES中的稳定 性，这对分析稳定性较差的物质具有重要意义. Dai 


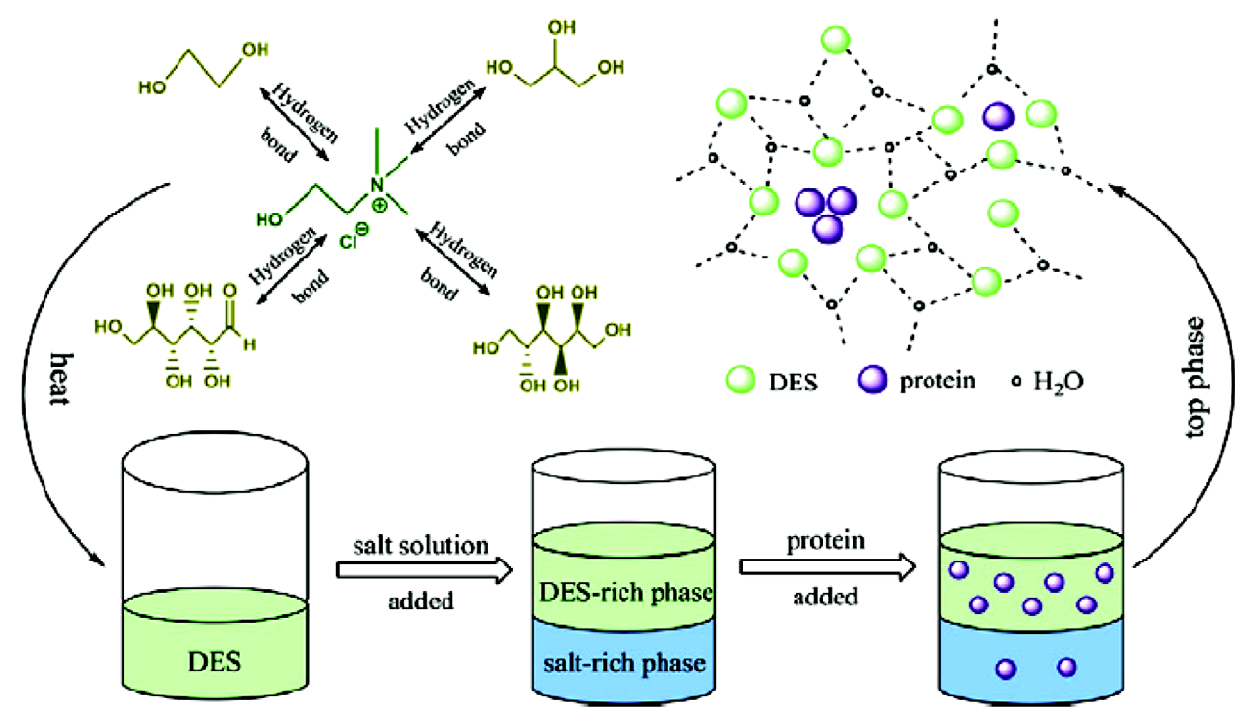

图 4 基于DES的双水相体系用于蛋白质的萃取(网络版彩图)

Figure 4 A DES-based aqueous two-phase system for protein extracting (color online).

等 ${ }^{[74]}$ 探究了从红花中萃取的天然色素在不同NADES 中的稳定性. 他们发现，花青素在葡萄糖-氯化胆碱和 蔗糖-氯化胆碱的NADES中比在酸性的NADES (脯氨 酸-苹果酸或乳酸-葡萄糖)中更稳定. NADES稳定能力 的提高可以通过减少水的含量, 增加其黏度实现. NADES这种较强的稳定能力或许是因为溶解的物质 与NADES之间较强的氢键相互作用. Dai 等 ${ }^{[75]}$ 合成了 一系列NADES，萃取长春花的花瓣中的花青素，研究 了NADES对花青素的萃取效率以及花青素在NADES 中的稳定性. 由乳酸和葡萄糖合成的NADES (LGH)以 及 1,2 -丙二醇和氯化胆碱合成的NADES $(\mathrm{PCH})$ 与传统 的有机溶剂相比具有相近的萃取能力, 然而花青素在 LGH中的稳定性是在酸性乙醇中的3倍, 有力地促进了 萃取和分析进程. 花青素在LGH中之所以如此稳定, 或 许是因为它与NADES组分间的较强的氢键相互作用. 该研究表明, NADES作为萃取溶剂, 黏度比极性具有 更重要的作用.

Tang 等 ${ }^{[76]}$ 将DESs作为萃取剂应用到顶空溶剂微 萃取方法中，萃取了日本扁柏叶子中的三种萜类化合 物并通过 GC进行分析. 所用的DES 是通过氯化胆碱和 乙二醇以不同的摩尔比合成. 用 $\mathrm{GC}$ 检测时, 不需要除 去DES. 相比于超声辅助的萃取, 该方法更简单快速.

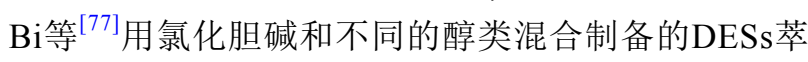
取日本扁柏中的两种黄酮类化合物. 各种因素被研究
来选择合适的DESs 和萃取方式, 其中氯化胆碱和 $1,4-$ 丁二醇以 $1: 5$ 的比例合成的DES对两种酮类化合物的 萃取效果最佳. 该方法与现存的方法相较, 对黄酮类物 质具有相似或更大的萃取量, 而且成本低、毒性低. $\mathrm{Nie}$ 等 $^{[78]}$ 则采用微波辅助DES萃取结合顶空固相微萃 取的方法分析了烟草中的挥发性化合物. 以氯化胆碱 和乙二醇 $(1: 3$, 摩尔比)合成的DES作为最适合的溶剂, 结合扫描电子显微镜图可以看出, 烟草样品在微波辐 射下采用DES处理后, DES可以破坏细胞壁, 从烟草细 胞中释放出更多的挥发性成分. 与传统的固相微萃取 方法相比，该方法简单高效，可以检测到更多的挥发 性成分.

Jeong 等 ${ }^{[79]}$ 设计合成了由丙三醇、 $L$-脯氨酸和蔗 糖(9:4:1) 3种组分的DES (GPS-5), 并将该DES作为萃 取剂结合超声辅助萃取的方法萃取了白参中极性的人 参㿝苷. 丙三醇的加入在降低DES黏性的同时提高了 萃取效率. 细胞实验表明, GPS-5 对萃取的人参㿝苷的 生物活性没有影响. 通过固相萃取(SPE)的方法, 乙醇 作为洗脱液, 可以回收被GPS-5 萃取的人参㿝苷, 具有 极好的回收率. 与之前萃取时间长且消耗大量有机试 剂的方法相比, 本实验中基于 GPS-5的萃取方法更加 有效.

Wang 等 ${ }^{[80]}$ 采用基于DES球磨机辅助萃取的方法 从丹参中简单快速 $(10 \mathrm{~s})$ 有效地萃取 3 种丹参酮类物 
质. 球磨机用来破坏细胞, 不同溶解度的丹参酮可以溶 解在具有不同黏度和极性的DESs中，其中氯化胆碱和 $1,2-$ 一二醇 $(1: 2$, 摩尔比)合成的DES具有最高的萃取效 率. 与传统的基于甲醇的超声辅助萃取相比, 该方法具 有快速、高效和环境友好的特点，可以用于不同样品 基质中多种天然产物的快速高效萃取，具有很大的潜 力. Chen等 ${ }^{[81]}$ 合成了 25 种基于氯化胆碱的DESs, 结合 微波辅助萃取的方法快速萃取了丹参中的 5 种活性成 分. 经过实验, 氯化胆碱和 1,2 -丙二醇 (1:1, 摩尔比)对目 标分析物具有最高产率. 在最优条件(溶剂: 含 $20 \%$ 水 的氯化胆碱-1,2-丙二醇; 微波功率: $800 \mathrm{~W}$; 温度: $70^{\circ} \mathrm{C}$; 时间： $11.11 \mathrm{~min}$ )下，亲水和疏水的物质可以同 时被萃取. 与传统的方法相比，该方法快速高效，可以 实现萃取的自动化(一批有 40 个样品). 缺点是设备花 费较高, 会萃取到许多干扰物, 在分析之前需要除杂. $\mathrm{Yu}$ 等 ${ }^{[82]}$ 则利用氯化胆碱和果糖 (3:2, 摩尔比)合成的 DES建立了基于DES的微波辅助水蒸气蒸馏提取黑胡 椒精油的方法, 并结合GC-MS分析其精油成分和含量. 与传统的水蒸气蒸馏提取相比, 该方法不仅能提取得 到更多的精油，而且精油中的化合物成分也明显增多. 此外，Yu等 ${ }^{[83]}$ 还利用氯化胆碱和乙二醇 (1:4, 摩尔比) 合成的DES用上述方法提取 3 种豆冦属中药中的精油, 并结合GC-MS对精油成分进行了分析. 与传统的方法 相比，该方法提取到的精油中的化合物成分明显增多， 而且在大多数精油中，碳氢化合物的相对含量也明显 提高. 由此表明, 精油含量的提高是由于细胞内化合 物的释放而不是精油的氧化.

Zhuang 等 ${ }^{[84]}$ 用DESs萃取了侧柏叶中的黄酮苷. 先 合成了 12 种基于氯化胆碱、甜菜碱和 $L-$ 脯氨酸的 DESs用于最初的篮选, 再通过单因素实验优化萃取条 件. 结果表明, 氯化胆碱与乙酰丙酸(1:2, 摩尔比)合成 的DES (含 $75 \%$ 的水)是最有效的溶剂, 可同时萃取极 性和非极性生物活性物质，萃取效率高于传统的溶剂. 另外，在DESs中水的含量对萃取效率有显著影响。通 过大孔树脂LX-38可以回收DESs中的目标物，取得了 令人满意的回收率(77.44\% 98.92\%).

$\mathrm{Li}$ 等 ${ }^{[85]}$ 合成了DES (用摩尔比为 $1: 2$ 的氯化胆碱和 丙三醇合成)修饰的分子印迹聚合物(DES-MIPs)、 DES修饰的非印迹聚合物(DES-NIPs，无模板)、分子 印迹聚合物(MIPs，无DES)和非印迹聚合物(NIPs，无 DES，无模板)，并将其分别用于从金银花中纯化绿原
酸. 通过实验比较发现, DES-MIPs用作SPE具有最高 的吸附能力, 这可能是因为分子印迹特异性识别作用 以及DES提高了亲和力、选择性和吸附能力.

此外, DESs在天然产物分析方面的应用还可参见 表 $1^{[86 \sim 88]}$.

\section{DES在石油化工分析中的应用}

酚类物质在农药、防腐剂、合成纤维、工程塑 料、染料等方面有着广泛用途, 是重要的化工原料和 化工中间体. 它们主要来源于石油产品、煤焦油和煤 液化产物，从这些油中分离出酚类物质有着重要意 $义^{[89]} . \mathrm{Gu}$ 等 ${ }^{[90]}$ 用卤盐和氢键受体以恰当的比例制备了 4 种不同的DESs, 并将其应用到液相微萃取中, 再借助 HPLC检测了原油中的酚类物质. 其中, 以氯化胆碱和 乙二醇 (1:3, 摩尔比)制备的DES因黏度较低取得了最 高的萃取效率. 用再生之后的DES对 3 种酚类物质进行 萃取, 取得了与新制备的DES相同的效果. 与传统的液 相微萃取相比, 该方法在超声的帮助下有效缩短了萃 取时间，同时说明了通过液相微萃取的方法DESs可以 从非极性或弱极性溶剂中萃取极性物质. Yao 等 ${ }^{[91]}$ 采 用两种基于季铵盐的两性离子(甜菜碱和左旋肉碱)与 苯酚形成DES，该DES与油不互溶从而可有效地分离 油性混合物中的苯酚. 结果表明, 两性离子与苯酚的 摩尔比极大地影响分离效率, 左旋肉碱与苯酚的摩尔 比为 0.4 时, 苯酚的萃取效率可达 $94.6 \%$; 温度对该萃取 影响很小, 可在室温下进行; 在DES中的苯酚可以使用 反溶剂回收, 甜菜碱和左旋肉碱也可以再利用, 使用 4 次后结构没有改变, 萃取效率没有明显减少. 该文还对 分离机理进行了研究, 表明甜菜碱和左旋肉碱是通过 氢键作用与苯酚形成DES. 与之前合成的DESs相比, 甜菜碱和左旋肉碱作为萃取剂更稳定，具有生物可降 解性，同时避免了氯离子的引入，不会造成设备的严 重腐蚀. 另外, Yao 等 ${ }^{[22]}$ 也利用甜菜碱和左旋肉碱可以 与苯酚形成DES, 萃取油里面的苯酚. 他们研究了在常 压不同温度下两个三元系统(甲苯 + 苯酚 + 甜菜碱和甲 苯+苯酚 + 左旋肉碱) 的相平衡. 研究表明, 存在 3 种相 区：液、液-液和液-液-固，分离主要发生在后面两个 相区. Zhang等 ${ }^{[93]}$ 和Ren等 ${ }^{[94]}$ 分别利用胆碱衍生物和氯 化胆碱对从模拟油中萃取酚类物质进行了研究，萃取 效率可达 $90 \%$ 以上. Tang等 ${ }^{[95]} 、 \mathrm{Lin}^{\text {等 }}{ }^{[96]}$ 和 $\mathrm{Hou}$ 等 ${ }^{[97]}$ 利 
用季铵盐从模拟油中萃取酚类物质，取得了令人满意 的萃取效率. $\mathrm{Ji}$ 等 ${ }^{[98,99]}$ 也研究了从油性混合物中分离 酚类物质。他们设计合成了 3 种咪唑基双阳离子离子 液体和3种三甲胺基双阳离子离子液体，分别通过与 酚类物质形成DESs，用于分离油性混合物中的酚类物 质，其达到的最高萃取效率分别为 $96.6 \%$ 和 $97.0 \%$. 研 究发现，尽管最初酚类化合物的浓度不同，但萃取后 的浓度保持一致。离子液体经多次回收利用后，对酚 类物质的萃取效率没有明显变化. 借助傅里叶变换红 外光谱(FT-IR)对萃取机理进行研究, 结果表明, 在离 子液体和苯酚之间存在氢键相互作用。该方法应用到 实际的煤焦油中也取得了令人满意的效果.

吲哚在产品(如香料、药物和外源激素)的生产中 起着重要的作用. $\mathrm{J}$ 等 ${ }^{[100]}$ 用吲哚和3种季铵盐(QASs) 形成的DESs有效地萃取了洗油中的吲哚. 3种QASs分 别为四乙基氯化铵(TEAC)、四丙基氯化铵(TPAC)和 三乙基甲基氯化铵( TEMAC), 萃取能力依次为 TEAC $>$ TPAC $>$ TEMAC，其中TPAC与吲哚形成的DES 对吲哚的萃取效率最高, 可达 $96.7 \%$, 且 5 min即可完 成萃取. 此外, DESs中的QASs可以重复使用，且萃取 效率没有明显降低. 利用FT-IR对萃取机理进行研究, 结果表明，在QAS和吲哚之间存在氢键相互作用。与 其他方法比较，该方法萃取效率高，避免使用无机酸 碱, QASs可回收利用, 环境友好.

燃料中的有机硫化物已成为严重环境污染的主要 来源之一. 燃料脱硫已成为前沿科学课题, 亟待解决. 研究表明，DESs因其合成原料便宜易得，合成过程简 单且环境友好以及较高的萃取脱硫效率等特点, 在燃 料脱硫方面具有较大潜力. $\mathrm{Li}$ 等 ${ }^{[101]}$ 设计合成了一系列 不同氢键给体和氢键受体的DESs 并成功地应用到石 油的萃取脱硫中. 该研究表明, 氢键受体对脱硫作用具 有更大的影响. 在最优条件下, 四甲基氯化铵与聚乙二
醇合成的DES在一次循环中萃取效率可达 $82.83 \%$ ，高 于传统的功能化的离子液体. 5次循环之后萃取效率 可达 $99.48 \%$. 通过对萃取机理的研究发现, DES和苯 并噻吩之间氢键的形成导致了较高的脱硫效率. Jiang 等 ${ }^{[102]}$ 则合成了一系列类似胆碱的DESs 用于原油脱硫. 该文研究了键连到氮原子上具有不同直烷基链的DES 对脱硫效率的影响，其中由 $N, N$-二甲基乙醇胺、氯代 十二烷与 $\mathrm{FeCl}_{3}$ 合成的DES具有最高的脱硫效率. 通过 两种苯环取代的DESs (分别由 $N, N$-二甲基乙醇胺、芐 基氯与 $\mathrm{FeCl}_{3}$ 和 $N$-甲基二乙醇胺、芐基氯与 $\mathrm{FeCl}_{3}$ 合成) 研究萃取机理发现, 在本实验中具有苯环的DES与二 苯并噻吩之间 $\mathrm{CH}-\pi$ 相互作用强于 $\pi-\pi$ 相互作用.

\section{7 总结与展望}

在样品前处理中, 选择恰当的萃取剂对于实现待 测物的良好萃取以及减小基质干扰至关重要. 近年来, DESs作为一种新兴的绿色溶剂已经广泛应用到食 品、环境和生物样品等的分析中. 表1中列举了除正文 以外近 5 年DESs在样品前处理中的应用. DESs代替传 统的有机萃取剂, 在各种萃取技术中表现出了许多优 势：操作简单、成本低、环境友好，提高了萃取效率 以及分析方法的选择性和灵敏度, 并且减少了对环境 和身体健康的影响.

在未来的分析技术发展中, DESs在样品前处理中 的应用将展现出广阔的应用前景. 对于DESs的研究主 要集中在以下 3 个方面: (1) 合成具有不同极性的DESs, 结合新颖的萃取技术, 分析不同复杂样品基质中的多 种分析物; (2) 将DESs作为萃取剂、吸附剂或改性剂, 结合萃取技术应用到实际样品中微量待测物的分析; (3) 提高不同萃取技术的选择性和分析性能, 帮助简化 萃取和分离过程.

\section{参考文献}

1 Zhang Q, De Oliveira Vigier K, Royer S, Jérôme F. Chem Soc Rev, 2012, 41: 7108-7146

2 Wei L, Fan YJ. Chem Bull, 2011, 74: 333-339 (in Chinese) [韦露, 樊友军. 化学通报, 2011, 74: 333-339]

3 Sun J, Jia Y. Sci Sin Chim, 2016, 46: 1317-1329 (in Chinese) [孙进贺, 贾永忠. 中国科学: 化学, 2016, 46: 1317-1329]

4 Imperato G, Eibler E, Niedermaier J, König B. Chem Commun, 2005, 856: 1170-1172

5 Gutierrez MC, Ferrer ML, Mateo CR, del Monte F. Langmuir, 2009, 25: 5509-5515

6 Ballantyne AD, Barker R, Dalgliesh RM, Ferreira VC, Hillman AR, Palin EJR, Sapstead R, Smith EL, Steinke NJ, Ryder KS. $J$ Electroanal 
Chem, 2018, 819: 511-523

7 Abbott AP, Ballantyne A, Harris RC, Juma JA, Ryder KS. Phys Chem Chem Phys, 2017, 19: 3219-3231

8 Wang L, Fan YJ, Wei L, Liu HX, Sun SG. J Electrochem, 2015, 21: 543-547 (in Chinese) [王莉, 校友军, 韦露, 刘海霞, 孙世刚. 电化学, 2015, 21: 543-547]

9 Pena-Pereira F, Kloskowski A, Namieśnik J. Green Chem, 2015, 17: 3687-3705

10 Rodríguez-Álvarez MJ, Vidal C, Díez J, García-Álvarez J. Chem Commun, 2014, 50: 12927-12929

11 Xiong X, Han Q, Shi L, Xiao S, Bi C. Chin J Org Chem, 2016, 36: 480-489 (in Chinese) [熊兴泉, 韩骞, 石霖, 肖上运, 毕成. 有机化学, 2016, 36: 480-489]

12 Gao G, Wang P, Liu P, Zhang W, Mo L, Zhang Z. Chin J Org Chem, 2018, 38: 846-854 (in Chinese) [高歌, 王萍, 刘鹏, 张卫红, 默丽萍, 张占 辉. 有机化学, 2018, 38: 846-854]

13 Raghuwanshi VS, Ochmann M, Hoell A, Polzer F, Rademann K. Langmuir, 2014, 30: 6038-6046 Procentese A, Johnson E, Orr V, Garruto Campanile A, Wood JA, Marzocchella A, Rehmann L. Bioresource Tech, 2015, 192: 31-36 Tan T, Qiao X, Wan Y, Qiu H. Chin J Chromatogr, 2015, 33: 934-937 (in Chinese) [谭婷, 乔釒金, 万益群, 邱洪灯. 色谱, 2015, 33: 934-937] Cunha SC, Fernandes JO. TrAC Trends Anal Chem, 2018, 105: 225-239 jsfa.9230 

2018, 36: 5-11]

51 Pirsaheb M, Fattahi N. RSC Adv, 2018, 8: 11412-11418

52 Zeng H, Qiao K, Li X, Yang M, Zhang S, Lu R, Li J, Gao H, Zhou W. J Sep Sci, 2017, 40: 4563-4570

53 Zarei AR, Nedaei M, Ghorbanian SA. J Mol Liquids, 2017, 246: 58-65

54 Zounr RA, Tuzen M, Khuhawar MY. J Mol Liquids, 2017, 242: 441-446

工, 2014, 34: 42-45]

90 Gu T, Zhang M, Tan T, Chen J, Li Z, Zhang Q, Qiu H. Chem Commun, 2014, 50: 11749-11752

91 Yao C, Hou Y, Ren S, Wu W, Zhang K, Ji Y, Liu H. Chem Eng J, 2017, 326: 620-626 
Yao C, Hou Y, Ren S, Ji Y, Wu W. Fluid Phase Equilib, 2017, 448: 116-122

93 Zhang Y, Li Z, Wang H, Xuan X, Wang J. Sep Purif Technol, 2016, 163: 310-318

94 Ren SH, Xiao Y, Wang YM, Kong J, Hou YC, Wu WZ. Fuel Process Technol, 2015, 137: 104-108

95 Tang W, Liu L, Li G, Zhu T, Row KH. Korean J Chem Eng, 2017, 34: 814-821

96 Lin Z, Hou Y, Ren S, Ji Y, Yao C, Niu M, Wu W. Fluid Phase Equilib, 2016, 429: 67-75

97 Hou Y, Kong J, Ren Y, Ren S, Wu W. Sep Purifi Technol, 2017, 174: 554-560

98 Ji Y, Hou Y, Ren S, Yao C, Wu W. Energy Fuels, 2017, 31: 10274-10282

99 Ji Y, Hou Y, Ren S, Yao C, Wu W. Fuel Process Technol, 2018, 171: 183-191

100 Ji Y, Hou Y, Ren S, Niu M, Yao C, Wu W. Fuel, 2018, 215: 330-338

101 Li C, Li D, Zou S, Li Z, Yin J, Wang A, Cui Y, Yao Z, Zhao Q. Green Chem, 2013, 15: 2793-2799

102

Jiang W, Dong L, Liu W, Guo T, Li H, Yin S, Zhu W, Li H. Chem Eng Process-Process Intensif, 2017, 115: 34-38

\title{
Application of deep eutectic solvents in sample preparation
}

\author{
Xiaofei Han ${ }^{1,2}$, Jia Chen ${ }^{1}$, Haijuan Zhang ${ }^{1}$, Zhan $\mathrm{Li}^{1}$, Hongdeng Qiu ${ }^{1 *}$ \\ ${ }^{1}$ CAS Key Laboratory of Chemistry of Northwestern Plant Resources and Key Laboratory for Natural Medicine of Gansu Province, Lanzhou Institute \\ of Chemical Physics, Chinese Academy of Sciences, Lanzhou 730000, China \\ ${ }^{2}$ University of Chinese Academy of Sciences, Beijing 100049, China \\ *Corresponding author (email: hdqiu@licp.cas.cn)
}

\begin{abstract}
In the chemical analysis process, in order to concentrate the trace analytes in complex samples and remove the matrix interference, sample preparation is necessary. It is vitally important to develop a simple, efficient, rapid and environmentally friendly sample preparation technique. Deep eutectic solvents (DESs) as a kind of emerging green solvent, have unique features such as low volatility, biodegradability, environmental friendliness, low cost and adjustable miscibility compared with the traditional organic solvents. DESs have been widely used in the sample preparation by combining with various extraction techniques such as ultrasound assisted extraction, microwave-assisted extraction, hollow fiber extraction and solid phase extraction. It can not only improve extraction efficiency and reduce the cost of conventional analysis, but also reduce the detrimental impacts on human health and environmental pollution. The present review provides the applications of DESs in the analysis of food, environment, biological samples, natural products and petrochemical industry in recent 5 years.
\end{abstract}

Keywords: deep eutectic solvents (DESs), sample preparation, extraction, chemical analysis

doi: $10.1360 / \mathrm{N} 032018-00192$ 